\title{
Treatment Approach to Coronavirus Disease (COVID-19) Seen Early After Open Heart Surgery. Case Report
}

\author{
Ersin Çelik ${ }^{1}$ (D) - Ahmet Rıfkı Çora ${ }^{1}$
}

Accepted: 23 June 2020 / Published online: 2 July 2020

(C) Springer Nature Switzerland AG 2020

\begin{abstract}
SARS-CoV-2 was reported for the first time in China on December 31,2019, as the cause of some pneumonia cases characterized by fever, cough, dyspnea, myalgia, and fatigue. Here, we present our approach to a 54-year-old male patient who had coronary artery bypass (CABG) surgery diagnosed as high probability coronavirus disease 2019 (COVID-19) in early postoperative period.
\end{abstract}

Keywords SARS-CoV-2 · Coronavirus disease 2019 • Coronary artery bypass surgery · Open heart surgery

\section{Introduction}

Severe acute respiratory syndrome coronavirus (SARS-CoV-2) has been reported for the first time in China on December 31, 2019, as the cause of some unknown pneumonia cases [1]. Within 2-14 days after exposure to the virus, some people develop coronavirus disease 2019 (COVID-19), characterized by fever, cough, dyspnea, myalgia, and fatigue. High mortality rates related to pneumonia, shock, acute respiratory distress syndrome, acute cardiac injury, and acute kidney injury were observed especially in patients with chronic disease and the elderly [2].

Although it has been a short time since it was reported for the first time, COVID-19 was declared as a pandemic by the World Health Organization on March 11, 2020 [3]. We aimed to present our approach to high probability COVID-19 pneumonia which developed on early postoperative period in our patient after coronary artery bypass grafting (CABG) operation, which was not reported in the literature before.

\section{Case Presentation}

The written consent form was obtained from the patient.

This article is part of the Topical Collection on Covid-19

Ersin Çelik

dr.ersincelik@gmail.com

1 Department of Cardiovascular Surgery, Isparta City Hospital, Sanayi Mahallesi Atatürk Bulvarı No: 51, 32200 Isparta, Turkey

\section{Patient Information}

A 54-year-old male patient presented as non-ST elevation myocardial infarction underwent emergent coronary angiography and critical stenosis evaluated on proximal left anterior descending artery (LAD) and the first diagonal branch. CABG surgery planned and taken to service follow-up. Routine blood tests performed on preoperative period were normal. On echocardiography, ejection fraction was $65 \%$. Seventy percent stenosis on the right and $60 \%$ stenosis on the left internal carotid artery were detected on routine carotid color Doppler ultrasonography. Pulmonary function tests and chest X-ray (Fig. 1a) were applied. After chest physician evaluation, dual inhaler medication was started. Following a week of inhaler treatment, patient's pulmonary function tests recovered and taken to the operation.

Median sternotomy was performed under general anesthesia. Two vessel CABG were applied by using left internal mammary artery and saphenous vein under cardiopulmonary bypass (CPB). Patient was taken to cardiovascular surgery intensive care unit uneventfully after the operation.

\section{Clinical Finding and Timeline}

Patient was extubated uneventfully and continued intensive care unit follow-up without any clinical problem. There was no pathology evaluated on chest X-ray taken on second postoperative day. (Fig. 1b). After removing the chest tubes and arterial lines, patient was taken to service follow-up. Results of the blood tests taken on 

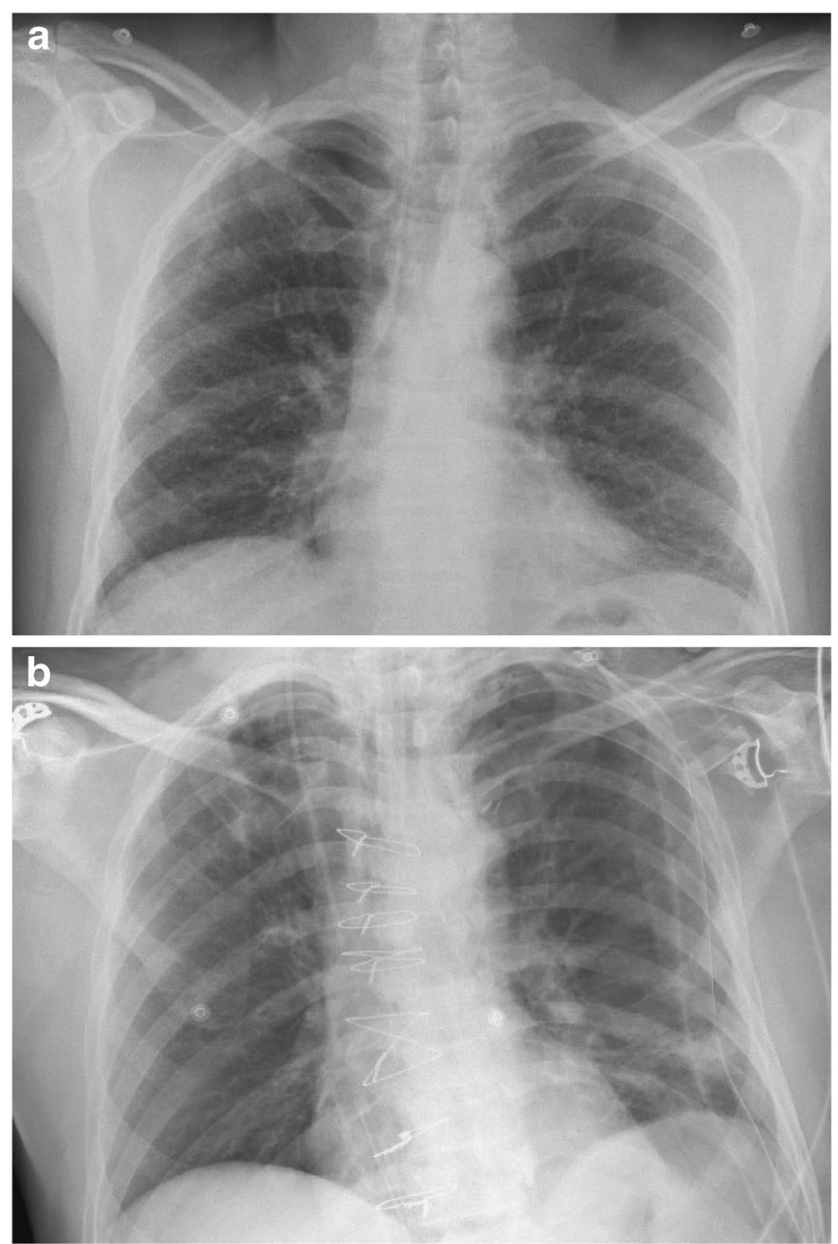

Fig. 1 a Preoperative lung X-ray. b Chest X-ray on the 2nd postoperative day

the 5th postoperative day were white blood cell (WBC) $18.11\left(10^{3} / \mu \mathrm{L}\right)$, lymphocyte $1.05\left(10^{3} / \mu \mathrm{L}\right)$, lymphocyte count $5.8 \%$, eosinophil $0.01\left(10^{3} / \mu \mathrm{L}\right)$, C-reactive protein (CRP) $17.5 \mathrm{mg} / \mathrm{dl}$, respectively. On physical examination, body temperature was raised to $38.2{ }^{\circ} \mathrm{C}$ on the 5 th postoperative day. Oxygen saturation in room air was 95\%, and heart rate and blood pressure were 90/ min and $110 / 60 \mathrm{mmHg}$, respectively.

\section{Diagnostic Assessment}

Control chest X-ray performed for evaluating the cause of this hyperthermia and infiltration areas were evaluated. Thorax computed tomography (CT) was taken for further investigation. Multifocal ground-glass opacities with peripheral and basal distribution, peripherally located bilateral infiltrative pneumonia, and vascular thickening were detected on thorax CT (Fig. 2).

CO-RADS 4 was reported on thorax CT of the patient. After consultations applied by chest physicians and infectious disease departments of our hospital, COVID-19 was evaluated as a high probability due to the laboratory tests, radiological findings, and clinical course. Patient was taken to the COVID19 isolation service; swabs were taken from the oropharynx and nasopharynx for real-time polymerase chain reaction (RTPCR) test.

\section{Therapeutic Intervention}

Since RT-PCR analysis results within $24-48 \mathrm{~h}$, the treatment of the patient was started with $2 \times 75 \mathrm{mg}$ oral oseltamivir phosphate tablet (Tamiflu, F. Hoffmann-La Roche Ltd., Basel, Switzerland); followed by $2 \times 400 \mathrm{mg}$ loading dose, with $2 \times 200 \mathrm{mg}$ oral hydroxychloroquine sulfate tablet (Plaquenil, Sanofi Synthelabo Limited, Fawdon, England); and followed by $1 \times 500 \mathrm{mg}$ loading dose, with $1 \times 250 \mathrm{mg}$ oral azithromycin tablet (Azitro, Deva Holding A.Ș., Tekirdağ, Turkey) without waiting for the results. This treatment protocol which is suggested by the Turkish Republic Ministry of Health and Covid-19 Science Committee is the first treatment step for whole patients.

\section{Follow-up and Outcomes}

Control RT-PCR test was sent $48 \mathrm{~h}$ after the first test and the first RT-PCR test was negative. The second test result was also negative. Fever control was achieved on the third day of the treatment. WBC and CRP values decreased. Infiltration areas regressed in control thorax CT (Fig. 3). On the fifth day of the therapy, treatment was completed. The patient was hemodynamically stable and had no respiratory distress. He was evaluated by the hospital pandemic team and recommended to be discharged and have isolation at home for 14 days. The patient stated that he had no complaints in the outpatient control on the 14th day.

\section{Discussion}

Although RT-PCR is accepted as the gold standard for the diagnosis of COVID-19, positivity is reported in $30-60 \%$ in series depending on the lack of experience on sampling, lack of cooperation of the patient, and transfer and kit performance [4]. And also according to reports, when disease manifests on the pulmonary area, viral load began to decrease, and for that reason, RT-PCR results could become negative. Therefore, thorax CT has become a widely used diagnostic feature for COVID-19 pneumonia. Studies comparing thorax CT and RT-PCR methods have been added to literature, and the sensitivity and specificity results of the radiological diagnosis are quite satisfactory [5]. The COVID working group of the Dutch Radiological Society has developed the CO-RADS 
Fig. 2 Postoperative thorax CT diagnosed as CO-RADS 4. a Bilateral emphysematous changes. b, c Peripherally located bilateral infiltrative pneumonia and vascular thickening. d Multifocal ground-glass opacities with peripheral and basal distribution
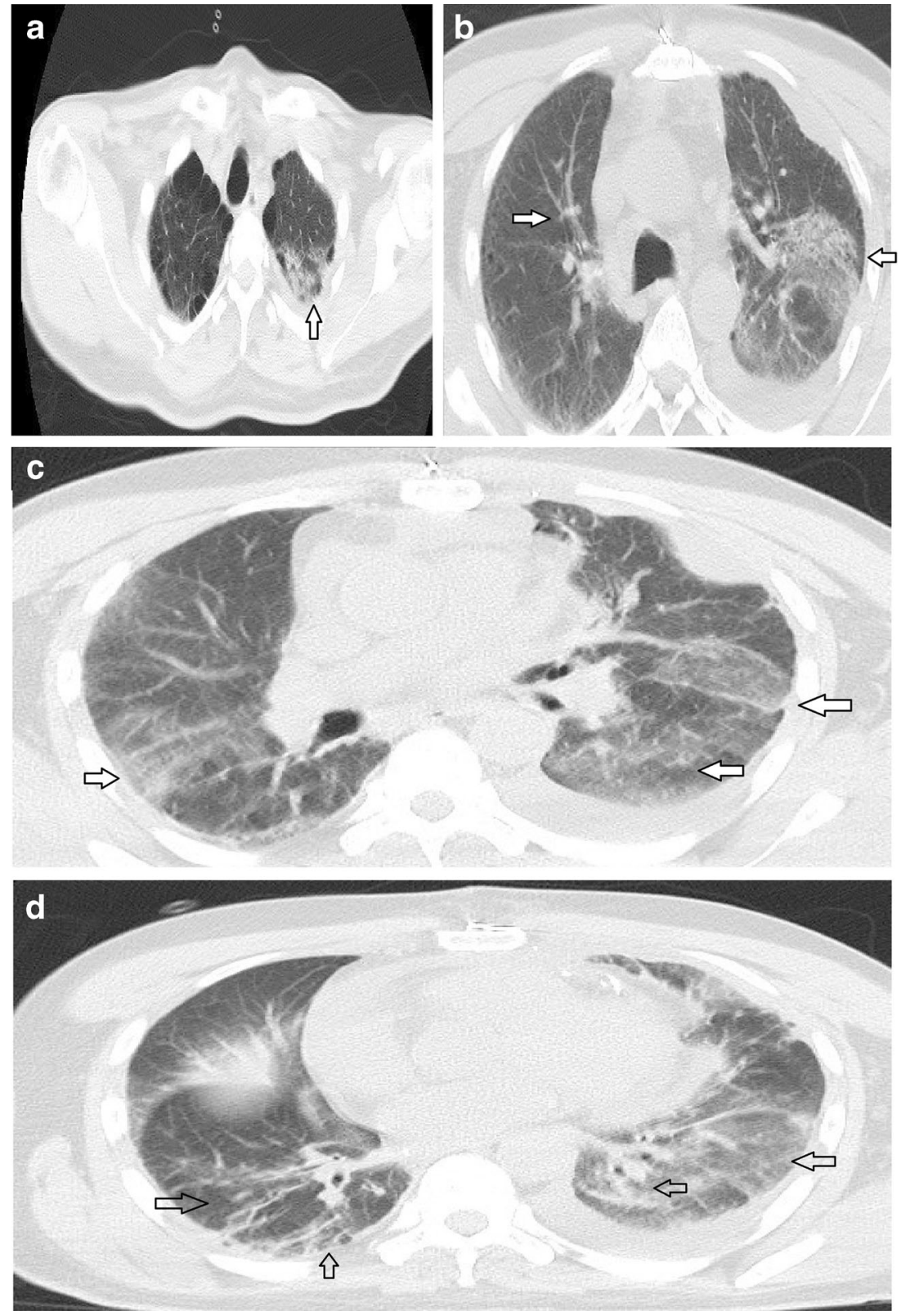

classification for thorax CT evaluation and taken it into clinical use [6].

When we evaluated our case according to the CORADS classification, we determined it as CO-RADS 4 (high probability COVID-19 pneumonia). On blood tests of patients, lymphocytopenia, eosinopenia, and decreased lymphocyte count were reported with COVID19 [7]. Eosinopenia and low lymphocyte count were detected in our case as well.

Systemic inflammatory response occurs as a result of cytokine release and complement system activation caused by CPB effect [8]. With the resultant immune system changes, these patients become more susceptible to possible infections. The patients that have undergone
CABG operation are generally those who are elderly and have multiple accompanying risk factors. Therefore, they are in a high-risk group in terms of morbidity and mortality that might be developed due to COVID-19. Having considered our patient as high risk, without waiting for the RT-PCR result, we started the specific treatment for COVID-19 immediately, by evaluating clinical, laboratory, and radiology findings. Although both RT-PCR tests were negative, we continued the treatment for 5 days. Because no physical and hemodynamic impairment was evaluated in our case, response to our therapy was followed up by the means of control thorax CT. After 5 days of the treatment, there was an obvious regression evaluated on thorax CT. 
Fig. 3 Control thorax CT on policlinic control
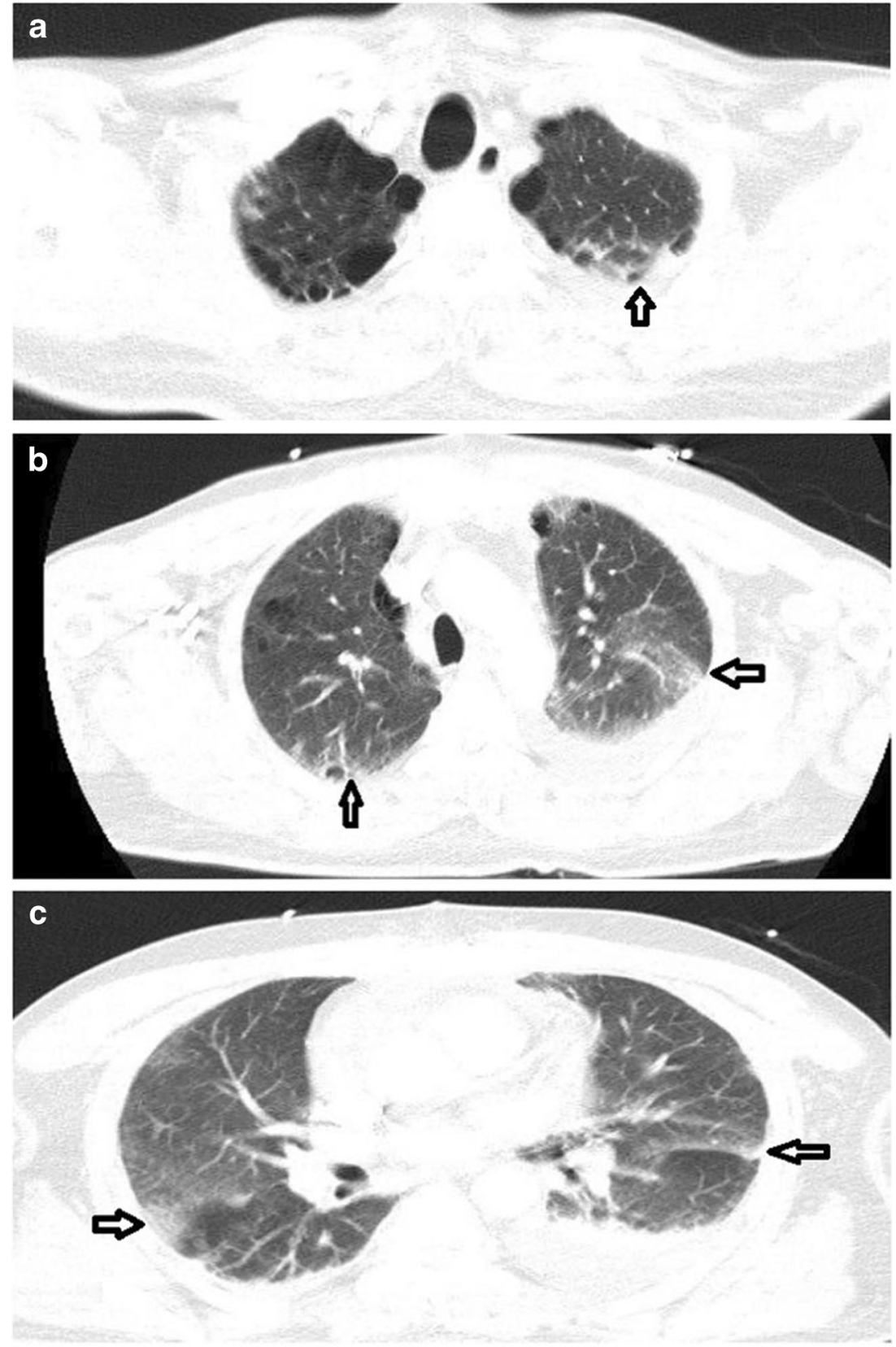

\section{Conclusion}

We should assume and keep in mind that there are also pandemic and asymptomatic carriers, especially in elderly patients who undergo open heart surgery with many additional risk factors. We believe that, in case of doubt, early treatment should not be ignored in the presence of clinical, laboratory, and radiological findings even if RT-PCR is negative.

Authors' Contributions All authors confirm in accordance with the Committee on Publication Ethics (COPE) guidelines and International Committee of Medical Journal Editors (ICMJE) criteria. Each author had made a substantial contribution to the conception and design, acquisition of data, and/or analysis and interpretation of data. Each author participated in the drafting of the article, revised it critically for important intellectual content, and had read before the approval of the final manuscript.

\section{Compliance with Ethical Standards}

Conflict of Interest The authors declare that they have no conflicts of interest.

Ethical Approval All procedures performed in studies involving human participants were in accordance with the ethical standards of the institutional and/or national research committee and with the 1964 Helsinki declaration and its later amendments or comparable ethical standards. 
Informed Consent Written informed consent for the publication was obtained from the patient.

\section{References}

1. World Health Organization. Novel coronavirus-China. Available at: (https://www.who.int/csr/don/12-january-2020-novel-coronaviruschina/en HYPERLINK "https://www.who.int/csr/don/12-january2020-novel-coronavirus-china/en/"/). Accessed 12 Jan 2020.

2. Huang C, Wang Y, Li X, Ren L, Zhao J, Hu Y, et al. Clinical features of patients infected with 2019 novel coronavirus in Wuhan, China. Lancet. 2020;395(10223):497-506.

3. World Health Organization. Rolling updates on coronavirus disease (COVID-19). Available at: (https://www.who.int/emergencies/ diseases/novel-coronavirus-2019/events-as-they-happen). Accessed 11 Mar 2020.

4. Yang Y, Yang M, Shen C, Wang F, Yuan J, Li J, et al. Evaluating the accuracy of different respiratory specimens in the laboratory diagnosis and monitoring the viral shedding off $2019-\mathrm{nCoV}$ infection. MedRxiv 2020 https://doi.org/10.1101/2020.02.11.20021493

5. Ai T, Yang Z, Hou H, Zhan C, Chen C, Lv W, et al. Correlation of chest CT and RT-PCR testing in coronavirus disease 2019 (COVID19) in China: a report of 1024 cases. Radiology. 2020 https://doi.org/ 10.1148/radiol.2020200642

6. Bai HX, Hsieh B, Xiong Z, Halsey K, Chai JW, Tran TML, et al. Performance of radiologists in differentiating COVID-19 from viral pneumonia on chest CT. Radiology. 2020 https://doi.org/10.1148/ radiol.2020200823

7. Lia F, Xu A, Zhang Y, Xuan W, Yan T, Pan K, et al. Patients of COVID-19 may benefit from sustained lopinavir-combined regimen and the increase of eosinophil may predict the outcome of COVID19 progression. Int J Infect Dis. 2020 https://doi.org/10.1016/j.ijid. 2020.03.013

8. Day JRS, Taylor KM. The systemic inflammatory response syndrome and cardiopulmonary bypass. Int J Surg. 2005;2(3):129-40.

Publisher's Note Springer Nature remains neutral with regard to jurisdictional claims in published maps and institutional affiliations. 